\title{
Antibody Response is Differentially Influenced by PLGA-PAD4 Particle Characteristics
}

\author{
Manish Manish, ${ }^{1,2,3}$ and Rakesh Bhatnagar ${ }^{1, *}$ \\ ${ }^{1}$ School of Biotechnology, Jawaharlal Nehru University, New Delhi, 110067, India \\ ${ }^{2}$ Department of Biosciences, Manipal University Jaipur, Jaipur-Ajmer Express Highway, Rajasthan 303007 \\ ${ }^{3}$ Lab no 406, National Institute of Malaria Research, Dwarka, Sec-8, New Delhi \\ "Corresponding author: Rakesh Bhatnagar, School of Biotechnology, Jawaharlal Nehru University, New Delhi, 110067, India. Tel: +91-1126704079, Fax: +91-1126717040, E-mail: \\ rakeshbhatnagar@jnu.ac.in
}

Received 2016 November 24; Revised 2017 January 03; Accepted 2017 January 03.

\begin{abstract}
Background: Anthrax, as a zoonotic disease, is considered a major bioterror threat. Development of effective prophylactic approaches is needed to combat this disease. The protective antigen domain 4 (PAD4) has been extensively studied as a vaccine candidate against anthrax. Previously, we showed that PAD4 can be successfully encapsulated in poly (lactide-co-glycolide) (PLGA)-based particulate systems.

Objectives: We aimed to determine if there is an intricate relationship between the parameters of PLGA particulate process and the associated immunogenicity.

Methods: In this study, PLGA (50:50) and PLGA (85:15) with a similar molecular weight were used as the polymer variants. Polymer variants were evaluated using 3 adaptations of w/o/w solvent evaporation method. Six variants of PLGA-PAD4 particulate systems were developed and studied for size, antigen content, and immunogenicity.

Results: $\mathrm{NaCl}$ in the external aqueous phase was required to achieve nonporous and spherical microparticles. Particle size was found to be dependent on the applied methods, and there was no significant difference in size by varying the PLGA compositions. PLGA variants showed a differential behavior with antigen encapsulation in the evaluated methods. The hydrophobic particulate vaccine with the mean diameter of $\sim 3 \mu$ m elicited the highest IgG titer, compared to particles with mean diameters of $\sim 0.2$ and $\sim 7 \mu \mathrm{m}$.
\end{abstract}

Conclusions: This study could help determine the composition of novel PAD4-encapsulated PLGA-based vaccine formulations.

Keywords: Anthrax, Vaccine, PLGA, Polymer Composition, Particle Size

\section{Background}

Anthrax is caused by a Gram-negative, nonmotile, rodshaped bacterium, known as Bacillus anthracis. There are uncertainties about prophylactic approaches against anthrax, such as multiple booster doses, toxicity, use of adjuvants, and stability. The protective antigen of $B$. anthracis is the principal component of all approved human vaccines against anthrax. However, this protective antigen has problems, such as stability and selection of suitable adjuvants. Overall, encapsulation of protective antigen in biodegradable polymers has not been successful, so far (1).

Protective antigen domain 4 (PAD4) is responsible for binding with the host anthrax toxin receptors, encoded by tumor endothelial marker-8 and capillary morphogenesis gene-2 receptors (2). The interaction of PAD4 with the host cell and monoclonal antibody has been studied in great detail (3-6). PAD4 has been evaluated in various vaccine formulations, such as plant-based (7) and alfalfa mosaic virusmediated expression systems (8). Moreover, it has been applied with rabies virus glycoprotein as a carrier protein (9), calreticulin as an adjuvant (10), and lethal factor as a fusion protein (11).

The crystal structure of isolated PAD4 has a structural conformation similar to PAD4 in native protective antigen molecules (12). PAD4 can maintain its structural integrity and bind with host cell receptors at an acidic $\mathrm{pH}$ (12). This property of PAD4 can be an advantage for poly (lactide-coglycolide) (PLGA)-based encapsulation, as an acidic $\mathrm{pH}$ environment is induced during PLGA degradation (13). This environment can cause protein aggregation, oxidation, deamidation, and acylation $(14,15)$. Also, PAD4 can be produced by recombinant protein expression, thus providing a safe and defined immunogen (16).

In our previous study, we showed that recombinant PAD4 can be successfully encapsulated in a PLGA-based particulate system $(17,18)$. However, there is an intricate relationship between the parameters of PLGA particulate process and immunogenicity. Surface porosity reduces the antigen content in PLGA particles (19). Overall, particle size has been considered as a major influencing parameter for

Copyright @ 2017, International Journal of Infection. This is an open-access article distributed under the terms of the Creative Commons Attribution-NonCommercial 4.0 International License (http://creativecommons.org/licenses/by-nc/4.0/) which permits copy and redistribute the material just in noncommercial usages, provided the original work is properly cited. 
immunogenicity. However, there are conflicting reports on the correlation of particle size with the resultant immune response (20). The polymer composition can change the hydrophobicity of polymer matrices (21); antigen dose has been also reported to influence the desired immune response (22).

With this background in mind, in this study, the process parameters and their effects on the elicited immunogenicity were studied. Three adaptations of w/o/w method were used with 2 compositional variants of PLGA. The particles were evaluated to achieve an optimized immunoglobulin G (IgG) response. The hydrophobic particulates with the mean diameter of $\sim 3 \mu$ m elicited the highest IgG titer among all the evaluated particle variants.

\section{Methods}

\subsection{Purification of Recombinant PAD4}

PAD4 was purified via Ni-NTA denaturing affinity chromatography with urea denaturation lysis on column renaturation, as described in the literature $(17,18)$. The bacterial pellets, used for the purification steps, were collected from a 4 L Escherichia coli culture; therefore, the antigen used for particle formulations was from the same batch. Also, 250 $\mathrm{mM} \mathrm{NaCl}$ was used in the final antigen stock buffer to prevent protein aggregation (12).

\subsection{Formulation of PLGA Nanoparticles/Microparticles}

Two polymer variants of PLGA (Sigma-Aldrich; P21915G lot \#051M1298V and 430471 lot \#MKBG1113V) were used in this study (Table 1). The polymers were of similar molecular weight and inherent viscosity, but of different monomer compositions. Also, polymer end-groups were ester-terminated.

Table 1. The PLGA polymer variants used in this study. Values within the brackets provide the lot-specific values. Table 1 shows that polymer variants used in this study only varied with respect to lactide content.

\begin{tabular}{lcc}
\hline Variants & PLGA 1 & PLGA 2 \\
\hline Composition & $50: 50(52: 48)$ & $85: 15$ \\
\hline Molecular weight & $40000-75000$ & $50000-75000$ \\
\hline Inherent viscosity, $\mathbf{d L} / \mathbf{g}$ & $0.55-0.75(0.61)$ & $0.55-0.75(0.65)$ \\
\hline Ester-terminated & Yes & Yes \\
\hline Form & Amorphous & Amorphous \\
Transition temperature, ${ }^{\circ} \mathbf{C}$ & $45-50$ & $45-50$ \\
\hline
\end{tabular}

PLGA particulates were prepared via 3 methodological adaptations of $w / 0 / w$ solvent evaporation method. In the first method, $200 \mathrm{mg}$ of PLGA was dissolved in $4 \mathrm{~mL}$ of dichloromethane, and a $100 \mu \mathrm{L}$ aliquot of concentrated recombinant PAD4 $(10 \mathrm{mg} / \mathrm{mL})$ was used as the internal aqueous phase. W/O emulsion was prepared using $2 \mathrm{~mm}$ of stepped microtip at 35\% amplitude for 60 seconds $(750 \mathrm{~W}$, Sonic Vibra-Cell ${ }^{\mathrm{TM}}$ sonicator).

An aqueous solution of $1 \%$ polyvinyl alcohol (PVA) was used as the external aqueous phase. The w/o/w emulsion was prepared, using $6 \mathrm{~mm}$ stepped tip at 30\% amplitude for 110 seconds. The emulsion was stirred for 6 hours for the evaporation of dichloromethane. Particles were harvested by centrifugation at 15,000 g for 15 minutes and washed 3 times with sterile deionized water.

Subsequently, the particles were suspended in $5 \mathrm{~mL}$ of sterile deionized water and frozen in liquid nitrogen to avoid phase separation. Particles were kept at $-80^{\circ} \mathrm{C}$ for 1 hour and then lyophilized at $-54^{\circ} \mathrm{C}$ and $0.003 \mathrm{mBar}$ for 18 hours. The PLGA particles, prepared with the first method, were in the nanometer size range. Particles prepared from PLGA(50:50) and PLGA(85:15) were termed as PAD4-NP1 and PAD4-NP2, respectively.

In the second method, the process parameters remained similar to method 1, except for the preparation of w/o/w emulsion via homogenization with an IKA homogenizer probe ( $\mathrm{S} 10 \mathrm{~N}-10 \mathrm{G})$ at speed of 2 for 10 minutes. The effect of $\mathrm{NaCl}$ on particle porosity was also explored by varying the concentration of $\mathrm{NaCl}$ in the external aqueous phase. The particles were harvested by centrifugation at $1500 \mathrm{~g}$ for 5 minutes. PLGA particles, prepared by method 2 , were in the micrometer size range. The particles prepared with PLGA (50:50) and PLGA (85:15) were termed as PAD4MP1 and PAD4-MP2, respectively.

In the third method, $352 \mathrm{mg}$ of PLGA was dissolved in $800 \mu \mathrm{L}$ of dichloromethane. The concentrated recombinant PAD4 $(10 \mathrm{mg} / \mathrm{mL})$ was used in $100 \mu \mathrm{L}$ aliquot as the internal aqueous phase. W/O emulsion was prepared via homogenization, using the IKA $10 \mathrm{~N}-5 \mathrm{G}$ probe at maximum speed for 30 seconds. Also, $800 \mu \mathrm{L}$ of $1 \%$ PVA was used as the external aqueous phase. The w/o/w emulsion was prepared via homogenization, using the IKA homogenizer probe (S10N-5G) at maximum speed for 30 seconds.

The $\mathrm{W} / \mathrm{O} / \mathrm{W}$ emulsion was transferred to $8 \mathrm{~mL}$ of $0.5 \%$ PVA and stirred for 2 hours to evaporate dichloromethane. The particles were harvested by centrifugation at $1500 \mathrm{~g}$ for 5 minutes. PLGA particles, prepared by method 3, were in the micrometer size range. The particles prepared using PLGA (50:50) and PLGA (85:15) were termed as PAD4-MP3 and PAD4-MP4, respectively.

\subsection{Characterization of Particle Morphology}

The morphological characteristics of PLGA particulates were evaluated, using a scanning electron microscope 
(Zeiss EVO40, Carl Zeiss, Thronwood, NY, USA) and a transmission electron microscope (JEM 2100F, Jeol Ltd., Tokyo, Japan), as described in the literature (Manish et al. 2013; Manish et al. 2016).

\subsection{Determination of Particle Size}

Particle size was measured by light scattering and polarization intensity differential scattering (PIDS) as a specific measure (LS ${ }^{\mathrm{TM}} 13320$ SW, Beckman Coulter Inc). Also, complete Mie theory was used as an optical model.

\subsection{Determination of Antigen Content}

The antigen content of PAD4 in PLGA particulate systems was evaluated, using micro-bicinchoninic acid assay (micro-BCA assay). Acetonitrile was used to dissolve PLGA and precipitate PAD4. The precipitated PAD4 was dissolved in $1 \%$ sodium dodecyl sulfate (SDS), as described in the literature $(17,18)$.

\subsection{Ethical Considerations}

Mice experiments were carried out under the regulations of the Institutional Animal Ethics Committee, Jawaharlal Nehru University, New Delhi, India. Inbred BALB/c mice were procured from the national institute of nutrition (NIN), Hyderabad, India. The mice were housed in individually ventilated animal cages in a pathogen-free, controlled environment.

\subsection{Evaluation of IgG Response}

The anti-PAD4 IgG response was evaluated, using enzyme-linked immunosorbent assay (ELISA), as described in the literature (Manish et al. 2013). Briefly, the wells were coated overnight with $100 \mu \mathrm{L}$ of PAD4 ( $5 \mathrm{mg} / \mathrm{mL}$ ). Individual serum samples were serially diluted in phosphatebuffered saline (PBS), assayed in triplicate alongside the serum from unimmunized mice, and incubated for 2 hours.

Horseradish peroxidase (HRP)-conjugated goat antimouse IgG (sc2005, Santa Cruz Biotechnology) was used as the secondary antibody. TMB substrate reagent (BD OptEIATM) was added as a colorimetric substrate and incubated for 30 minutes. The cutoff point was calculated for every serial dilution with a confidence interval of $99 \%$ for increased specificity (Frey et al. 1998). A higher confidence interval could reduce sensitivity and decrease the risk of overtitration. The endpoint titer was calculated as the reciprocal value of the highest dilution, having an absorbance above the cutoff point.

\subsection{Statistical Analysis}

The statistical significance of particle size, antigen content, and immune response was measured by one-way ANOVA and Turkey's multiple-comparison test.

\section{Results}

PAD4 was purified via Ni-NTA affinity chromatography. The w/o/w solvent evaporation method was employed for the preparation of PLGA particles. In the w/o/w solvent evaporation method, the antigen, which needs to be encapsulated, is dissolved in the internal aqueous phase. Therefore, to achieve a higher antigen content in PLGA particles, a higher concentration of antigen in the internal aqueous phase is required. In this study, we used PAD4 at a concentration of $10 \mathrm{mg} / \mathrm{mL}$. However, recombinant proteins frequently aggregate/precipitate at higher concentrations (23). NaCl has been reported to reduce the aggregation of PAD4 (12). Consequently, $250 \mathrm{mM} \mathrm{NaCl}$ was used in the final PAD4 stock buffer to maintain the uniformity of the solution.

3.1. Sodium Chloride in the External Aqueous Phase Was Required to Achieve Nonporous and Spherical Microparticles

PAD4-NP1 and PAD4-NP2 were prepared using method 1 , and the size of particles was in the nanometer range. In method 1 , the ratio of organic phase to the internal aqueous phase was 40:1, whereas the dispersed-to-continuous phase ratio was 1:3. First, emulsion was prepared via sonication at $35 \%$ amplitude for 60 seconds. The second emulsion was prepared via sonication at 30\% amplitude for 110 seconds. The formulated nanoparticles were nonporous and spherical (Figure 1).

PAD4MP-1 and PAD4-MP2 were prepared using method 2 , and the size of particles was in the micrometer range. In this method, the ratio of organic phase to internal aqueous phase was 40:1, whereas dispersed-to-continuous phase ratio was 1:3. The first emulsion was prepared by sonication at $35 \%$ amplitude for 60 seconds, while the second emulsion was prepared via homogenization for 10 minutes. The formulated microspheres showed a porous morphology (Figure $1 \mathrm{~B}$ and Figure $1 \mathrm{C}$ and supplementary file, appendix 1 ).

In the first and second methods, similar polymer concentrations, organic solvents, internal aqueous phases, and external aqueous phases were used. Particles in the nanometer range were of smooth surface characteristics, whereas particles in the micrometer range had porous surface features. Formation of pores on the particle surface is reported as a complex phenomenon (Giteau et al. 2008); however, in our study, the porosity of PLGA particles was 

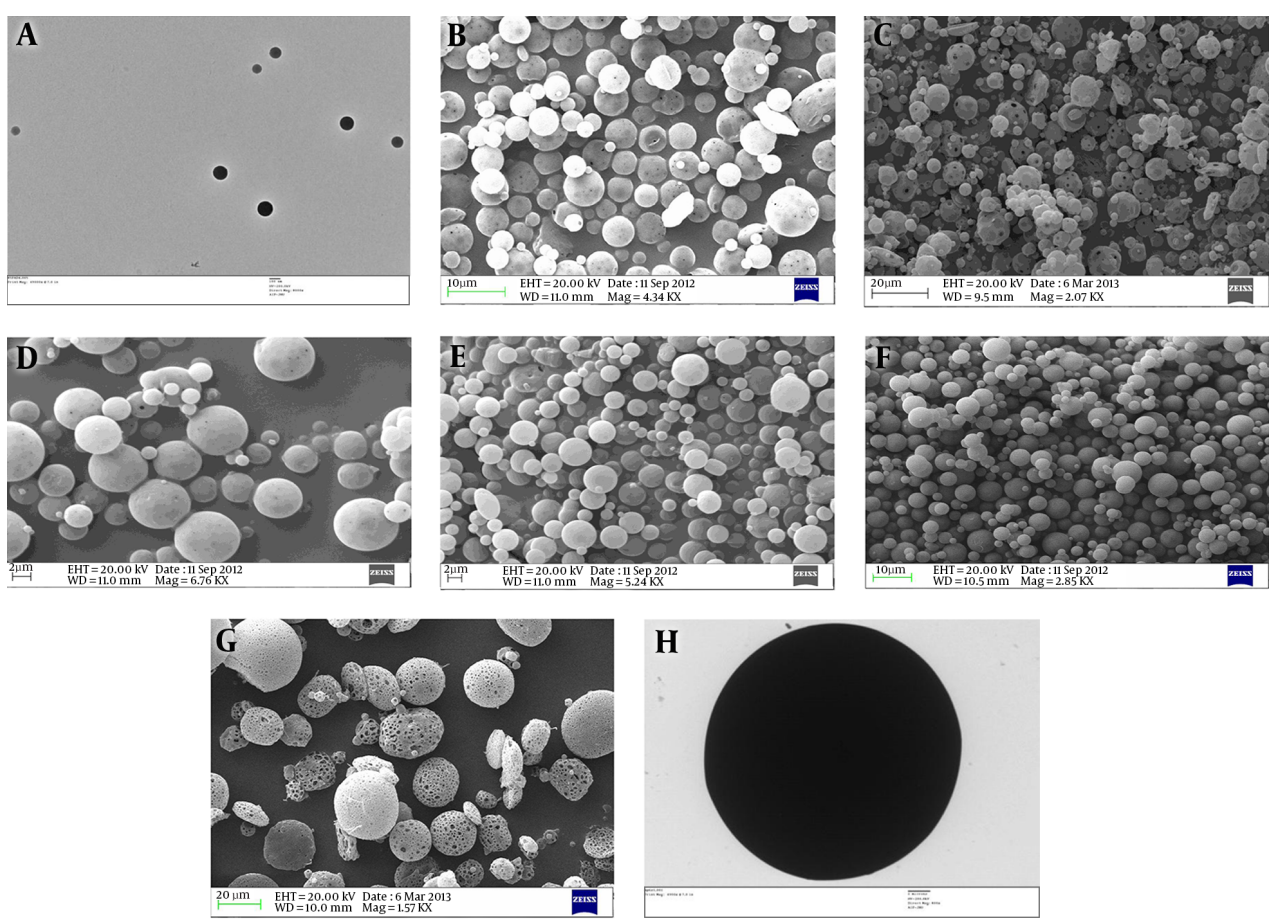

A, Transmission electron micrograph of PAD4-NP2. This figure shows that PAD4-NP2s are in the nanometer size range and spherical with a smooth surface. B, Scanning electron micrograph of PAD4-MP1. This figure shows that PAD4-MP1s are in a micrometer size range and have a porous morphology. C, Scanning electron micrograph of PAD4-MP2. This figure shows that PAD4-MP2s are in a micrometer size range and have a porous morphology. D, Scanning electron micrograph of PAD4-MP1. PAD4-MP1 was prepared using 1\% $\mathrm{NaCl}$ in the external aqueous phase. This figure shows reduction in the porosity of PAD4-MP1. E, Scanning electron micrograph of PAD4-MP1. PAD4-MP1 was prepared using 2\% $\mathrm{NaCl}$ in the external aqueous phase. This figure shows reduction in PAD4-MP1 porosity. F, Scanning electron micrograph of PAD4-MP1. PAD4-MP1 was prepared using 3\% NaCl in the external aqueous phase. This figure shows that PAD4-MP1s are in the micrometer size range and spherical, with a smooth surface. G, Scanning electron micrograph of PAD4-MP3. This figure shows that PAD4-MP3s are in a micrometer size range and have a porous morphology. H, Transmission electron micrograph of PAD4-MP3. PAD4-MP3 was prepared using $1 \% \mathrm{NaCl}$ in the external aqueous phase. This figure shows that PAD4-MP3 is in a micrometer size range and spherical with a smooth surface.

associated with increased particle size (comparison of Figure $1 \mathrm{~A}$ with Figure $1 \mathrm{~B}$ and Figure $1 \mathrm{C}$ and supplementary file, appendix 1). The porosity of particle surface was associated with both hydrophilic (PLGA 50:50) and hydrophobic (PLGA 85:15) polymers (Figure 1B and Figure 1C).

$\mathrm{NaCl}$ in the external aqueous phase has been reported to achieve a nonporous particle formulation (De Rosa et al. 2003). Therefore, $\mathrm{NaCl}$ concentration gradient in the external aqueous phase was employed for PAD4-MP1 and PAD4-MP2 formulations. PAD4-MP1 was prepared with $1 \%$ $\mathrm{NaCl}$ (Figure 1D), 2\% $\mathrm{NaCl}$ (Figure 1E), and 3\% $\mathrm{NaCl}$ (Figure $1 \mathrm{~F})$. It was observed that the increased $\mathrm{NaCl}$ concentration reduced the surface porosity of PAD4-MP1 (Figure 1D and E E and F).F).

$\mathrm{NaCl}$ gradient in the external aqueous phase was also employed for PAD4-MP2. PAD4-MP2 was formulated in 1\% $\mathrm{NaCl}$ (supplementary file, appendix 2), $2 \% \mathrm{NaCl}$ (supplementary file, appendix 3), and $3 \% \mathrm{NaCl}$ (supplementary file, appendix 4). It was observed that the increased $\mathrm{NaCl}$ con- centration reduced the surface porosity of PAD4-MP2 (supplementary file, appendixes 2,3 , and 4 ).

It was speculated that pore formation could be a result of low PLGA concentration or sonication force used for the formulation of PAD4-MP1 and PAD4-MP2. Therefore, high concentration of PLGA and mechanical force in form of homogenization were used for the formulation of PAD4-MP3 and PAD4-MP4. However, surface porosity was observed in PAD4-MP3 (Figure 1G and supplementary file, appendix 5) and PAD4-MP4 (supplementary file, appendix 6). Even in method 3, surface porosity was associated with both hydrophilic and hydrophobic polymers (Figure $1 G$ and supplementary file, appendix 6). The addition of $\mathrm{NaCl}$ to the external aqueous phase reduced the porosity of PAD4-MP3 (Figure $1 \mathrm{H}$ ) and PAD4-MP4 (supplementary file, appendix 7). 
3.2. The Explored Methods for the Preparation of PLGA Particulates Produced Particles of Single-Peak Population with a Nanometer or Micrometer Size Range

Particles PAD4-NP1, PAD4-NP2, PAD4-MP1 (prepared in 3\% NaCl), PAD4-MP2 (prepared in 3\% NaCl), PAD4-MP3 (prepared in $1 \% \mathrm{NaCl}$ ), and PAD4-MP4 (prepared in $2 \% \mathrm{NaCl}$ ) were evaluated for population size characteristics. Particle size has been considered as a major influencing factor for the desired immune response; therefore, the explored methods were examined for particle population distribution.

Method 1 produced particles in the nanometer range with a single-peak particle population. The particle diameter varied from approximately $50 \mathrm{~nm}$ to $500 \mathrm{~nm}$. Method 2 also produced particles with a single-peak particle population; however, the particles were in the micrometer range. Particle diameter varied from approximately $1.5 \mu \mathrm{m}$ to 10 $\mu \mathrm{m}$. The particle populations, formulated by methods 1 and 2, showed distinct distributions with no overlap (Figure 2).

Method 3 also produced particles with a single-peak particle population. The particle diameter varied from approximately $3.5 \mu \mathrm{m}$ to $30 \mu \mathrm{m}$. There was no overlap between the particle populations formulated by methods 1 and 3. However, the particle population formulated by methods 2 and 3 overlapped, although the overlap was less than $20 \%$ (Figure 2).

Figure 2. The Overlap Diagram of Particle Population Distributions in the Explore Methods

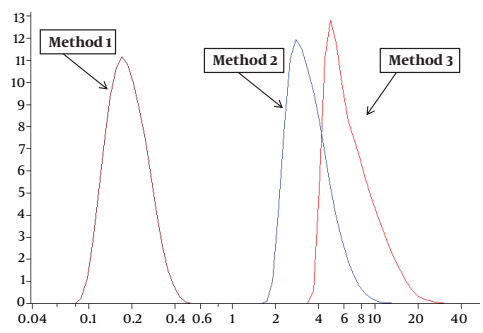

The graph was drawn by LS 13320 software (Beckman Coulter, Inc). This figure shows that particle populations, formulated by methods 1 and 2, do not overlap. The particle populations, formulated by methods 1 and 3, also do not overlap. Particle populations, formulated by methods 2 and 3, overlap, although it is less than $20 \%$.

The mean diameter of PAD4-NP1 was $0.230 \mu \mathrm{m}$, whereas the mean diameter of PAD4-NP2 was $0.193 \mu \mathrm{m}$ (Table 2). There was no significant difference between the polymer variants with a nanometer size range. Also, the mean diameter of PAD4-MP1 was $3.66 \mu \mathrm{m}$, whereas the mean diameter of PAD4-MP2 was $2.96 \mu \mathrm{m}$. In methods 1 and 2, similar polymer concentrations, organic solvents, internal aqueous phases, and external aqueous phases were used.
Table 2. Size of PLGA particulates. The particle size was measured by light scattering and polarization intensity differential scattering (PIDS) as a specific measure ( $\mathrm{LS}^{\mathrm{TM}} 13$ 320 SW, Beckman Coulter, Inc). Complete Mie theory was used as the optical model. The results are presented as mean \pm standard deviation(SD) of 3 independent samples.

\begin{tabular}{ll}
\hline PLGA Particulate systems & $\begin{array}{l}\text { Arithmetic Mean Diameter (Mean } \pm \text { SD of 3 } \\
\text { Independent Measurements), } \mu \mathbf{m}\end{array}$ \\
\hline PAD4NP1 (50:50) & $0.230 \pm 0.084$ \\
\hline PAD4NP2 (85:15) & $0.193 \pm 0.068$ \\
\hline PAD4MP1 (50:50) & $3.66 \pm 1.45$ \\
\hline PAD4MP2 (85:15) & $2.96 \pm 1.43$ \\
\hline PAD4MP3 (50:50) & $7.21 \pm 3.16$ \\
\hline PAD4MP4 (85:15) & $6.86 \pm 5.49$ \\
\hline
\end{tabular}

The only difference between methods 1 and 2 was the applied mechanical force. Therefore, the mean diameter of particles varied mainly due to the applied mechanical forces. The difference between the mean diameters of PAD4-MP1 and PAD4-MP2 was not significant; consequently, PLGA polymer variants did not influence the particle size. The mean diameter of PAD4-MP3 was $7.21 \mu \mathrm{m}$, whereas the mean diameter of PAD4-MP4 was $6.86 \mu \mathrm{m}$. The effect of polymer variant on particle size was not observed in method 3.

Methods 1 and 2 showed a narrow-peak particle population distribution, whereas in method 3 , the particle diameter varied from 3.5 to $30 \mu \mathrm{m}$. In method 3 , the polymer concentration was increased to $440 \mathrm{mg} / \mathrm{mL}$, whereas in methods 1 and 2, the polymer concentration was $50 \mathrm{mg} / \mathrm{mL}$. In method 3, volume of the organic phase was $800 \mu \mathrm{L}$, which is 5 times less than the volume of the organic phase, used in methods 1 and 2 . These factors constitutively enhanced the viscosity of the organic phase in method 3.

A viscous solution was needed to increase the size of particle formulation. However, viscous solutions are difficult to emulsify, which can contribute to the wide particle population distribution observed in method 3, although maximum homogenization speed was employed. As our objective was to delineate the effect of particle parameters on PAD4 immunogenicity, we mainly concentrated on particles with a mean diameter in nanometer or up to $10 \mu \mathrm{m}$. It has been reported that particles greater than $10 \mu \mathrm{m}$ induce a low humoral antibody response (19). However, the influence of particle size on immunogenicity was not clearly elucidated from nanometer to $10-\mu \mathrm{m}$ particle populations (20). As the particle sizes were in the desired range, we evaluated the antigen content in PLGA particulates. 
3.3. Antigen Content in PLGA Particulates Varied with PLGA Composition and Applied Methods

The antigen was extracted from PLGA particulates and measured using micro-BCA assay. The antigen content in $10 \mathrm{mg}$ of PAD4-NP1 was $50 \mu \mathrm{g}$, whereas the antigen content in $10 \mathrm{mg}$ of PAD4-NP2 was $38.22 \mu \mathrm{g}$ (Table 3). Therefore, a significant change in antigen encapsulation was observed by varying the PLGA composition. Hydrophilic polymer showed a greater antigen encapsulation than hydrophobic polymer in method 1.

Table 3. The encapsulated antigens in PLGA particulate systems. The antigen content in lyophilized PLGA particulates was measured using micro-BCA assay after antigen extraction with acetonitrile and solubilization of antigen precipitate with $1 \%$ SDS. The results are presented as mean \pm standard deviation (SD).

\begin{tabular}{|ll}
\hline PLGA Particulate Systems & $\begin{array}{l}\text { Encapsulated Antigen per 10 mg of } \\
\text { Lyophilized PLGA Particulates, } \mu \mathrm{g}\end{array}$ \\
\hline PAD4-NP1 (50:50) & $50 \pm 0.68$ \\
\hline PAD4-NP2 (85:15) & $38.22 \pm 0.7$ \\
\hline PAD4-MP1 (50:50) & $37.18 \pm 0.65$ \\
\hline PAD4-MP2 (85:15) & $58.31 \pm 0.86$ \\
\hline PAD4-MP3 (50:50) & $33.87 \pm 0.96$ \\
\hline PAD4-MP4 (85:15) & $30.68 \pm 0.76$ \\
\hline
\end{tabular}

The antigen content in $10 \mathrm{mg}$ of PAD4-MP1 was 37.18 $\mu \mathrm{g}$, whereas the antigen content in $10 \mathrm{mg}$ of PAD4-MP2 was $58.31 \mu \mathrm{g}$ (Table 3). Even in method 2, a significant change in antigen encapsulation was observed by varying the PLGA composition. However, in method 2, the hydrophobic polymer showed a greater antigen encapsulation than the hydrophilic polymer. Therefore, the polymer variants showed a differential behavior with antigen encapsulation, which depended on particle size.

The antigen content in $10 \mathrm{mg}$ of PAD4-MP3 was 33.87 $\mu \mathrm{g}$, whereas the antigen content in $10 \mathrm{mg}$ of PAD4-MP4 was $30.68 \mu \mathrm{g}$ (Table 3). The antigen content in particles formulated by method 3 was less than that of methods 1 and 2 . In method 3, a higher concentration of PLGA was used, i.e., 352 mg of PLGA was dissolved in $800 \mu \mathrm{L}$ of dichloromethane. Consequently, a reduction was observed in antigen content per $10 \mathrm{mg}$ of dried PLGA particulates. In this study, antigen content was evaluated as encapsulated antigen per $10 \mathrm{mg}$ of dried PLGA particulates. As PLGA particulates are known to act as an antigen depot and a controlled delivery system, the antigen encapsulated in the particle can be a better correlate for immunogenicity than the antigen encapsulated in the overall formulation process (22).
3.4. PAD4-MP2 Elicited the Highest anti-PAD4 IgG Titer Among the Evaluated PLGA Particulates

The immunization study was carried out on inbred BALB/c mice; the number of mice in each group was 5 . The mice were immunized with $100 \mu \mathrm{g}$ of encapsulated antigen in form of PLGA particulates. The control group received $50 \mu \mathrm{g}$ of PAD4 alone. The anti-PAD4 IgG response was measured, using an adjuvant, single-dose immunization schedule. A group of mice was also immunized with blank PLGA particles; however, no anti-PAD4 IgG response was detected in this group.

The mice, only immunized with PAD4, showed mean anti-PAD4 IgG titers of 853.3 and 533.3 on days 14 and 28, respectively. The mean IgG titers in PAD4-NP1-immunized mice were 10,500 and 82,973 on days 14 and 28, respectively. Also, the mean IgG titers in PAD4-NP2-immunized mice were 8833 and 24,000 on days 14 and 28, respectively. The mean IgG titer in PAD4-MP1-immunized mice was 36,000 and 485,840 on days 14 and days 28 , respectively.

Moreover, the mean IgG titers in PAD4-MP2immunized mice were 59,480 and 980,680 on days 14 and 28, respectively. The mean IgG titers in PAD4-MP3immunized mice were 15,220 and 30,580 on days 14 and 28, respectively. Also, the mean IgG titers in PAD4-MP4 immunized mice were 44,000 and 188,000 on days 14 and 28, respectively (Figure 3).

Figure 3. PAD4-MP2 Elicited the Highest anti-PAD4 IgG Titer

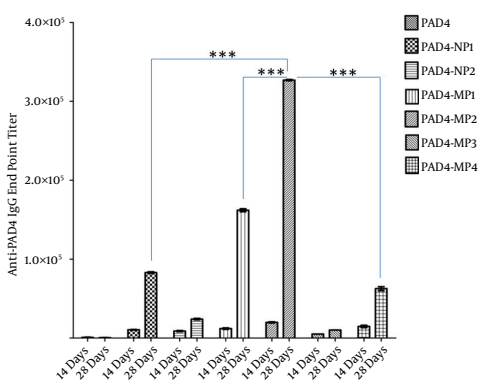

The sera from immunized mice $(n=5)$ were collected on days 14 and 28 postimmunization. PAD4-MP2 elicited the highest level of IgG titer among the evaluated PLGA particulates. Antibody titers (Y-axis) are presented on a linear scale. Error bars indicate \pm standard error (SE) of 3 experiments. ${ }^{* * *}$ denotes a statistically sig nificant change $(\mathrm{P}<0.001)$, as evaluated by one-way ANOVA and Tukey's multiplecomparison test.

PAD4 (in the absence of adjuvants with single-dose immunization) did not elicit a significant IgG titer. However, PAD4-PLGA particulates elicited a robust anti-PAD4 IgG response. PAD4-NP2 induced the lowest antibody titer among the evaluated particulates. IgG titer in PAD4NP2-immunized mice was approximately 40 times higher than the titer elicited in mice immunized with only PAD4. 
Therefore, enhancement of IgG titer with particulate formulation was clearly observed, compared to antigen alone.

Among PLGA particulates, PAD4-MP2 elicited the greatest IgG response. Particles with the mean diameter of $\sim 3$ $\mu \mathrm{m}$ (i.e., PAD4-MP1 and PAD4-MP2) elicited the highest antibody titer, compared to particles with the mean diameters of $\sim 0.2 \mu \mathrm{m}$ (i.e., PAD4-NP1 and PAD4-NP2) and $7 \mu \mathrm{m}$ (ie, PAD4-MP3 and PAD4-MP4). The hydrophilic polymers elicited the highest IgG titer in nanometer size range particles. In the micrometer size range, particles from the hydrophobic polymer elicited the greatest IgG response. The antigen content in particles was positively correlated with the immune response (Figure 3 ).

\section{Discussion}

Development of recombinant proteins leads to the identification of numerous recombinant antigens. However, use of antigens as vaccine candidates is limited by issues, such as fragile structure, proteolytic degradation, and booster doses. Previously, we showed that PAD4 could be successfully encapsulated in PLGA-based particulate systems. However, the effect of process parameters on particle characteristics and elicitation of IgG response was not clear. This study focused on the evaluation of PLGA-PAD4 variants for enhanced IgG response.

Addition of salt is often required to stabilize the protein solution (24). Particle porosity has been attributed to high osmotic pressure inside the internal aqueous phase (25). Addition of $\mathrm{NaCl}$ to the external phase seems to reduce the osmotic pressure difference between the internal and external aqueous phases $(25,26)$. Particles with a porous surface lead to an enhanced initial burst release. Initial burst release has been considered as a major drawback of PLGA-based particulate vaccine formulations (27).

The pores on the particle surface can reduce the antigen encapsulation (19). Particle porosity is also known to influence the antigen release rate (28). The biodegradation of porous PLGA particulates is reported to be faster than nonporous PLGA particulates (29). Therefore, porous particles can have pharmaceutical disadvantages due to substantial antigen release in the initial burst phase. Loss of antigen in porous particle formulations reduces its economical viability. Furthermore, great initial antigen release from porous particles reduces the efficacy of PLGA particulates to provide a long-term antigen release system (30). Therefore, we obtained nonporous PLGA particulates for the evaluation of particle characteristics and the associated immune response.

We observed the influence of PLGA composition variants on the antigen content of the formulated particles. In the study of rifampicin-loaded microsphere (size, $2 \mu \mathrm{m}$ ), the loading efficiency decreased in PLGA polymers with a high molecular weight. The reason could be the interaction of terminal carboxyl groups of PLGA with rifampicin. At higher molecular weights, the PLGA composition tends to influence the loading efficiency, while at lower molecular weights, the difference in PLGA composition does not influence the loading efficiency (31). As we evaluated the PLGA composition variants of high molecular weight, differential encapsulation of PAD4 in PLGA particles could be explained. It has been also reported that PLGA composition of 75:25 has a higher loading efficiency than PLGA composition of 50:50 for hepatitis B surface antigen-loaded particles with a size range of $2-4 \mu \mathrm{m}$ (32).

In the present study, the greatest IgG response was observed after immunization with PLGA polymer (85:15; mean diameter $\sim 3 \mu \mathrm{m}$ ). In the study of PLGA encapsulated hepatitis B surface antigen, the hydrophobic polymer (PLGA, 75:25) elicited a better IgG response than the hydrophilic polymer (PLGA, 50:50)(32). The chemical composition of polymers does not influence the extent of phagocytosis by antigen-presenting cells (33). However, antigen release from particulate systems is dependent on the chemical composition of polymer (21). The antigen release profile is also dependent on the antigen content of particles (21).

PLGA particles with a diameter smaller than $10 \mu \mathrm{m}$ are reported to be more immunogenic than particles with a diameter more than $10 \mu \mathrm{m}$ (19). This phenomenon can be attributed to the phagocytic activity of antigen-presenting cells. In the study of phagocytosis of PLGA microparticles, loaded with anti-tuberculosis rifampicin (diameter, 1 - 10 $\mu \mathrm{m}$ ) by alveolar macrophages, a considerable proportion of macrophages were not able to phagocytose $1-\mu \mathrm{m}$ particles. The phagocytosis was greater with 1 and $3 \mu \mathrm{m}$ particles than 6 and $10 \mu \mathrm{m}$ particles (34).

In the study of phagocytosis of albumin-loaded polystyrene particles by alveolar macrophages, internalization of particles with a diameter of $500 \mathrm{~nm}$ was 10 times more than particles with a diameter of $1 \mu \mathrm{m}$ and 100 times higher than 2 - $3 \mu \mathrm{m}$ particles (35). Therefore, differential interaction of particles with antigen-presenting cells can be speculated, based on the size of PLGA particulates. The polymer degradation rate of particles is not influenced by size. In the study of BSA-encapsulated PLGA particles with sizes of $0.1,1$, and $10 \mu \mathrm{m}$, the polymer degradation rate did not significantly vary (36).

With respect to the discussed issues, it can be inferred that PLGA composition variants modulate the immune response via differential antigen release, whereas particle size variants modulate the immune response via differential phagocytosis. Therefore, in the present study, we evaluated the composition and size variants to achieve opti- 
mum immune response.

In the present study, we observed a greater elicitation of IgG with microparticles, compared to nanoparticles. The higher elicitation of IgG with microparticles has been also reported for PLGA-encapsulated hepatitis B surface antigen (37). Also, an increase in IgG response has been observed by increasing the size of particles. By the comparison of 200, 500, and $1000 \mathrm{~nm}$ PLGA particles, encapsulating bovine serum albumin, $1000 \mathrm{~nm}$ particles elicited the highest IgG titer (38).

The immune response obtained in 500-nm particles was similar to $200-\mathrm{nm}$ particles in the evaluated immunization routes, i.e., subcutaneous, oral, and intranasal. The IgG1/IgG2a ratio did not change during the evaluation of particulate antigens, which suggests that particles with these size ranges follow the same antigen presentation pathway (38). However, an optimum size range is required for the greater elicitation of IgG response, as in the study of hepatitis B surface antigen, 5- $\mu \mathrm{m}$ particles showed greater immunogenicity than $12-\mu$ m particles (19).

\subsection{Conclusion}

We evaluated 6 variants of PLGA-encapsulated PAD4. The results could help achieve nonporous PLGA-PAD4 variants with a robust IgG response and design novel vaccines against anthrax. However, antigen release and phagocytosis also depend on the surface charge of particles; this factor was not considered in the current study. In the formulation parameters, only 3 methods were evaluated. However, statistically validated experimental designs, such as the Plackett-Burman design (39), are needed to delineate the effects of formulation parameters at low resolutions.

\section{Supplementary Material}

Supplementary material(s) is available here.

\section{Acknowledgments}

The present study was funded in part by the department of Biotechnology (DBT), Indian council of Medical research, and department of Science and technology (DST), India. MM is the recipient of Utrecht University short-stay fellowship, Fulbright fellowship, and SBRI-India GID fellowship (NIH research grant No., D43 TW000924, funded by the Fogarty international Center). We acknowledge the Beckman coulter Laboratory, Mumbai, India for their assistance in particle size measurements.

\section{Footnote}

Financial Disclosure: The authors declare no conflicts of interest.

\section{References}

1. Flick-Smith HC, Eyles JE, Hebdon R, Waters EL, Beedham RJ, Stagg TJ, et al. Mucosal or parenteral administration of microsphereassociated Bacillus anthracis protective antigen protects against anthrax infection in mice. Infect Immun. 2002;70(4):2022-8. doi: 10.1128/IAI.70.4.2022-2028.2002. [PubMed: 11895967].

2. Scobie HM, Rainey GJ, Bradley KA, Young JA. Human capillary morphogenesis protein 2 functions as an anthrax toxin receptor. Proc Natl Acad Sci U S A. 2003;100(9):5170-4. doi: 10.1073/pnas.0431098100. [PubMed: 12700348].

3. Leysath CE, Monzingo AF, Maynard JA, Barnett J, Georgiou G, Iverson $\mathrm{BL}$, et al. Crystal structure of the engineered neutralizing antibody M18 complexed to domain 4 of the anthrax protective antigen. J Mo Biol. 2009;387(3):680-93. doi: 10.1016/j.jmb.2009.02.003. [PubMed: 19361425].

4. Brossier F, Levy M, Landier A, Lafaye P, Mock M. Functional analysis of Bacillus anthracis protective antigen by using neutralizing monoclonal antibodies. Infect Immun. 2004;72(11):6313-7. doi: 10.1128/IAI.72.11.6313-6317.2004. [PubMed: 15501759].

5. Brossier F, Sirard JC, Guidi-Rontani C, Duflot E, Mock M. Functional analysis of the carboxy-terminal domain of Bacillus anthracis protective antigen. Infect Immun. 1999;67(2):964-7. [PubMed: 9916116].

6. Rosovitz MJ, Schuck P, Varughese M, Chopra AP, Mehra V, Singh Y, et al. Alanine-scanning mutations in domain 4 of anthrax toxin protective antigen reveal residues important for binding to the cellular receptor and to a neutralizing monoclonal antibody. $J$ Biol Chem. 2003;278(33):30936-44. doi:10.1074/jbc.M301154200. [PubMed: 12771151].

7. Gorantala J, Grover S, Goel D, Rahi A, Jayadev Magani SK, Chandra S, et al. A plant based protective antigen [PA(dIV)] vaccine expressed in chloroplasts demonstrates protective immunity in mice against anthrax. Vaccine. 2011;29(27):4521-33. doi: 10.1016/j.vaccine.2011.03.082. [PubMed: 21504775].

8. Brodzik R, Bandurska K, Deka D, Golovkin M, Koprowski H. Advances in alfalfa mosaic virus-mediated expression of anthrax antigen in planta. Biochem Biophys Res Commun. 2005;338(2):717-22. doi: 10.1016/j.bbrc.2005.09.196. [PubMed: 16236249].

9. Smith ME, Koser M, Xiao S, Siler C, McGettigan JP, Calkins C, et al. Rabies virus glycoprotein as a carrier for anthrax protective antigen. $\mathrm{Vi}$ rology. 2006;353(2):344-56. doi: 10.1016/j.virol.2006.05.010. [PubMed: 16820183].

10. Park YS, Lee JH, Hung CF, Wu TC, Kim TW. Enhancement of antibody responses to Bacillus anthracis protective antigen domain IV by use of calreticulin as a chimeric molecular adjuvant. Infect Immun. 2008;76(5):1952-9. doi: 10.1128/IAI.01722-07. [PubMed: 18285494].

11. Baillie LW, Huwar TB, Moore S, Mellado-Sanchez G, Rodriguez L, Neeson BN, et al. An anthrax subunit vaccine candidate based on protective regions of Bacillus anthracis protective antigen and lethal factor. Vaccine. 2010;28(41):6740-8. doi: 10.1016/j.vaccine.2010.07.075. [PubMed: 20691267].

12. Williams AS, Lovell S, Anbanandam A, El-Chami R, Bann JG. Domain 4 of the anthrax protective antigen maintains structure and binding to the host receptor CMG2 at low pH.Protein Sci. 2009;18(11):2277-86. doi 10.1002/pro.238. [PubMed: 19722284].

13. van de Weert $M$, Hennink WE, Jiskoot W. Protein instability in poly(lactic-co-glycolic acid) microparticles. Pharm Res. 2000;17(10):1159-67. doi: 10.1023/A:1026498209874. [PubMed 11145219]. 
14. Cleland JL, Powell MF, Shire SJ. The development of stable protein formulations: a close look at protein aggregation, deamidation, and oxidation. Crit Rev Ther Drug Carrier Syst. 1993;10(4):307-77. [PubMed: 8124728].

15. Houchin ML, Topp EM. Chemical degradation of peptides and proteins in PLGA: a review of reactions and mechanisms. J Pharm Sci. 2008;97(7):2395-404. doi: 10.1002/jps.21176. [PubMed: 17828756].

16. Perrie Y, Mohammed AR, Kirby DJ, McNeil SE, Bramwell VW. Vaccine adjuvant systems: enhancing the efficacy of subunit protein antigens. Int J Pharm. 2008;364(2):272-80. doi: 10.1016/j.ijpharm.2008.04.036. [PubMed: 18555624].

17. Manish M, Bhatnagar R, Singh S. Preparation and Characterization of PLGA Encapsulated Protective Antigen Domain 4 Nanoformulation. Methods Mol Biol. 2016;1404:669-81. doi:10.1007/978-1-4939-3389-1_43. [PubMed: 27076329].

18. Manish M, Rahi A, Kaur M, Bhatnagar R, Singh S. A single-dose PLGA encapsulated protective antigen domain 4 nanoformulation protects mice against Bacillus anthracis spore challenge. PLoS One. 2013;8(4):e61885. doi: 10.1371/journal.pone.0061885. [PubMed 23637922].

19. Thomas C, Gupta V, Ahsan F. Particle size influences the immune response produced by hepatitis B vaccine formulated in inhalable particles. Pharm Res. 2010;27(5):905-19. doi: 10.1007/s11095-010-0094-x. [PubMed: 20232117].

20. Oyewumi MO, Kumar A, Cui Z. Nano-microparticles as immune adjuvants: correlating particle sizes and the resultant immune responses. Expert Rev Vaccines. 2010;9(9):1095-107. doi:10.1586/erv.10.89. [PubMed: 20822351].

21. O'Hagan DT, Jeffery H, Davis SS. The preparation and characterization ofpoly(lactide-co-glycolide) microparticles: III. Microparticle/polymer degradation rates and the in vitro release of a model protein. Int J Pharm. 1994;103(1):37-45. doi:10.1016/0378-5173(94)90201-1.

22. Katare YK, Muthukumaran T, Panda AK. Influence of particle size, antigen load, dose and additional adjuvant on the immune response from antigen loaded PLA microparticles. Int J Pharm. 2005;301(12):149-60. doi: 10.1016/j.ijpharm.2005.05.028. [PubMed:16023313].

23. Bondos SE, Bicknell A. Detection and prevention of protein aggregation before, during, and after purification. Anal Biochem. 2003;316(2):223-31. doi: 10.1016/S0003-2697(03)00059-9. [PubMed: 12711344].

24. Pistel KF, Kissel T. Effects of salt addition on the microencapsulation of proteins using $\mathrm{W} / \mathrm{O} / \mathrm{W}$ double emulsion technique. J Microencapsul. 2000;17(4):467-83. doi: 10.1080/026520400405723. [PubMed 10898087].

25. De Rosa G, Quaglia F, Bochot A, Ungaro F, Fattal E. Long-term release and improved intracellular penetration of oligonucleotidepolyethylenimine complexes entrapped in biodegradable microspheres. Biomacromolecules. 2003;4(3):529-36. doi: 10.1021/bm025684c. [PubMed: 12741766].

26. Gomes dos Santos AL, Bochot A, Doyle A, Tsapis N, Siepmann J, Siepmann F, et al. Sustained release of nanosized complexes of polyethylenimine and anti-TGF-beta 2 oligonucleotide improves the outcome of glaucoma surgery. J Control Release. 2006;112(3):369-81. doi:10.1016/j.jconrel.2006.02.010. [PubMed: 16644054].
27. Yeo Y, Park K. Control of encapsulation efficiency and initial burst in polymeric microparticle systems. Arch Pharm Res. 2004;27(1):1-12. doi: 10.1007/BF02980037. [PubMed: 14969330].

28. Ghaderi R, Sturesson C, Carlfors J. Effect of preparative parameters on the characteristics of poly d,l-lactide-co-glycolide)microspheres made by the double emulsion method. Int J Pharm. 1996;141(1-2):20516. doi: 10.1016/0378-5173(96)04639-x.

29. Khang G, Cho JC, Lee JW, Rhee JM, Lee HB. Preparation and characterization of Japanese encephalitis virus vaccine loaded poly(L-lactideco-glycolide) microspheres for oral immunization. Biomed Mater Eng. 1999;9(1):49-59. [PubMed:10436853].

30. Huang X, Brazel CS. On the importance and mechanisms of burst release in matrix-controlled drug delivery systems. J Control Release. 2001;73(2-3):121-36. doi: 10.1016/S0168-3659(01)00248-6. [PubMed: 11516493].

31. Makino K, Nakajima T, Shikamura M, Ito F, Ando S, Kochi C, et al. Efficient intracellular delivery of rifampicin to alveolar macrophages using rifampicin-loaded PLGA microspheres: effects of molecular weight and composition of PLGA on release of rifampicin. Colloids Surf B Biointerfaces. 2004;36(1):35-42. doi: 10.1016/j.colsurfb.2004.03.018. [PubMed: 15261021].

32. Feng L, Qi XR, Zhou XJ, Maitani Y, Wang SC, Jiang Y, et al. Pharmaceutical and immunological evaluation of a single-dose hepatitis $\mathrm{B}$ vaccine using PLGA microspheres.J Control Release. 2006;112(1):35-42. doi: 10.1016/j.jconrel.2006.01.012. [PubMed: 16516999].

33. Tabata Y, Ikada Y. Macrophage phagocytosis of biodegradable microspheres composed of L-lactic acid/glycolic acid homoand copolymers. J Biomed Mater Res. 1988;22(10):837-58. doi: 10.1002/jbm.820221002. [PubMed: 3220838].

34. Hirota $\mathrm{K}$, Hasegawa $\mathrm{T}$, Hinata $\mathrm{H}$, Ito $\mathrm{F}$, Inagawa $\mathrm{H}$, Kochi $\mathrm{C}$, et al. Optimum conditions for efficient phagocytosis of rifampicinloaded PLGA microspheres by alveolar macrophages. J Control Release. 2007;119(1):69-76. doi: 10.1016/j.jconrel.2007.01.013. [PubMed: 17335927].

35. Foster KA, Yazdanian M, Audus KL. Microparticulate uptake mechanisms of in-vitro cell culture models of the respiratory epithelium. J Pharm Pharmacol. 2001;53(1):57-66. doi: 10.1211/0022357011775190. [PubMed: 11206193].

36. Panyam J, Dali MM, Sahoo SK, Ma W, Chakravarthi SS, Amidon GL, et al. Polymer degradation and in vitro release of a model protein from poly(D,L-lactide-co-glycolide) nano- and microparticles. J Control Release. 2003;92(1-2):173-87. [PubMed:14499195].

37. Kanchan V, Panda AK. Interactions of antigen-loaded polylactide particles with macrophages and their correlation with the immune response. Biomaterials. 2007;28(35):5344-57. doi: 10.1016/j.biomaterials.2007.08.015. [PubMed: 17825905].

38. Gutierro I, Hernandez RM, Igartua M, Gascon AR, Pedraz JL. Size dependent immune response after subcutaneous, oral and intranasal administration of BSA loaded nanospheres. Vaccine. 2002;21(1-2):6777. doi:10.1016/S0264-410X(02)00435-8. [PubMed: 12443664].

39. Rahman Z, Zidan AS, Habib MJ, Khan MA. Understanding the quality of protein loaded PLGA nanoparticles variability by Plackett-Burman design. Int J Pharm. 2010;389(1-2):186-94. doi: 10.1016/j.ijpharm.2009.12.040. [PubMed: 20038446]. 